\title{
CONCEPTO DE ESPIRITUALIDAD DEL EQUIPO MULTIDISCIPLINARIO DE UNA UNIDAD DE CUIDADOS PALIATIVOS: UN ESTUDIO DESCRIPTIVO
}

\author{
THE CONCEPT OF SPIRITUALITY IN A MULTIDISCIPLINARY TEAM FROM A \\ PALLIATIVE CARE UNIT: A DESCRIPTIVE STUDY
}

\author{
Patricia E. Sandoval Guzmán, Nancy E. Rangel Domínguez, Silvia R. Allende Pérez y \\ Leticia Ascencio Huertas
}

Unidad de Cuidados Paliativos del Instituto Nacional de Cancerología de México, México

Resumen

La espiritualidad, es una necesidad fundamental en el cuidado paliativo, sin embargo, la literatura reporta que existen inconsistencias en la definición de los términos "espiritualidad" y "religiosidad", lo que genera confusión en su abordaje con los pacientes. El presente estudio fue exploratorio, con el objetivo de conocer la concepción de espiritualidad y religiosidad que tiene un equipo multidisciplinario en una unidad de cuidados paliativos oncológicos. El estudio fue no experimental, transversal y descriptivo; con 34 profesionales de la salud. Para la obtención del concepto se empleó la técnica de redes semánticas naturales, utilizando dos palabras estímulo: Espiritualidad y Religiosidad. Dentro de los resultados se encontraron semejanzas entre ambas palabras, al ser definidos como: fe, dios, creencia, amor, religión, esperanza, creencias. Sin embargo, se presentan diferencias en el concepto de espiritualidad; definida como paz, alma, tranquilidad, armonía, meditación, bondad, espíritu; y trascendencia; mientras que el concepto de religiosidad; fue definida como iglesia, ritos, fanatismo, espiritualidad, compromiso, oración, reglas, y sacerdote. Concluyendo que los conceptos resultan confusos para el equipo de cuidados paliativos, sin embargo, cuentan con recursos personales para identificar las necesi-
Abstract

Spirituality is a fundamental need in palliative care; however, the literature reports that there are inconsistencies in the definition of the terms "spirituality" and "religiosity," which creates confusion in how to approach these issues with the patients. The present study was exploratory, with the aim to better understand the concept of spirituality and religiosity that a multidisciplinary team has in an oncology palliative care unit. The study was not experimental, cross-sectional and descriptive, with 34 health professionals. To obtain each concept, the natural semantic networks technique was used; using two stimulus words: spirituality and religiosity. Among the results, similarities between the two concepts were found, being both defined as faith, god, belief, love, religion, hope. However, there are differences that distinguish the concept of spirituality; defined as peace, soul, tranquility, harmony, meditation, kindness, spirit, and transcendence; from the concept of religiosity; defined as church, rituals, bigotry, spirituality, commitment, prayer, rules, and priest. Concluding that the concepts are confusing to the palliative care team, however they have personal resources to identify the spiritual needs although they lack of information and formal training to address this.

Correspondencia:

Psic. Patricia Eugenia Sandoval Guzmán

Instituto Nacional de Cancerología

Av. San Fernando, No. 22, Col. Sección XVI, Deleg. Tlalpan, C.P. 14700.

México, D.F., Mexico

E-mail: ptysand@gmail.com 
dades espirituales aunque carecen de información y entrenamiento formal para su abordaje.

Palabras Clave: Espiritualidad, religiosidad, profesionales de la salud, cuidados paliativos.
Key words: Spirituality, religiosity, health care providers, palliative care.

\section{INTRODUCCIÓN}

La espiritualidad es un tema de valioso y de creciente interés dentro del área de la salud. Al respecto se han realizado una serie de investigaciones que aportan evidencia que la coloca como una necesidad de atención de los pacientes, ya que entre otras cosas, impacta sobre variables como las conductas de cuidado de la salud y la calidad de vida ${ }^{(1-9)}$. En el contexto de los cuidados paliativos el tema de las necesidades espirituales de los pacientes cobra aun mayor importancia ${ }^{(5,9,10)}$ ya que, constituyen una de las áreas de atención en pacientes y familias que se enfrentan a enfermedades graves, tal como lo reconoce la Organización Mundial de la Salud ${ }^{(11)}$. Sin embargo, no se cuenta con estrategias formales para su evaluación y manejo, ya que se priorizan las necesidades físicas de los pacientes ${ }^{(2,9,12-15)}$ debido a que estos manifiestan con mayor frecuencia $y$ facilidad síntomas como el dolor físico u otras molestias, pero rara vez externan sus necesidades espirituales ${ }^{(2,7,14,16)}$. Esta es la realidad cotidiana en las unidades de cuidados paliativos, en la que tanto los pacientes como sus familias se ven constantemente confrontados con la muerte, lo que puede motivar la necesidad de abordar no sólo el sufrimiento físico. Las necesidades espirituales representan, entonces, un reto para los profesionales de la salud que atienden a pacientes en fases avanzadas de enfermedad $(2,5,7,16,17)$.

Conocer y entender las necesidades espirituales de los pacientes es un hecho que involucra al profesional de la salud desde diferentes sentidos: (a) En su práctica la- boral, ya que se requiere que el personal realice una búsqueda activa de las mismas en el momento de la evaluación del paciente, y que posteriormente las incorpore a la intervención. (b) En su actuar personal, ya que requiere aumentar la conciencia de la proximidad de la muerte ${ }^{(2,8,13,15,18,19)}$.

Con respecto a los profesionales de salud y espiritualidad, se han realizado estudios que analizan la postura personal y ejercicio de los profesionales de la salud, ante las necesidades espirituales de sus pacientes. Se ha encontrado que esta población admite la necesidad de la atención de los aspectos espirituales; de igual forma se reconoce a la espiritualidad como un recurso del paciente ante la situación de enfermedad y/o la muer$t^{(4,5,8,12,13,19,20)}$. Además se reporta que algunos profesionales de la salud, consideran que la atención de estas necesidades debería ser proporcionada por personas especializadas ${ }^{(1,5,13)}$.

Así mismo, los estudios reportan que el personal de salud identifica barreras para la atención espiritual de los pacientes, entre ellas se cuentan: la formación exclusivamente biomédica del clínico, en la que se consideran a los aspectos espirituales como poco relevantes; un entrenamiento inadecuado para la identificación de estas necesidades, dudas sobre su incorporación a la intervención, la ausencia de una postura u orientación espiritual en la vida de algunos profesionales; así como la ignorancia de los efectos positivos de la espiritualidad sobre la $\operatorname{salud}^{(5,8,12,13,21)}$.

Probablemente la principal limitación para la incorporación del estudio y ma- 
nejo de las necesidades espirituales de los pacientes por parte del personal de salud, radique en la conceptualización que estos últimos tengan de la espiritualidad, ya que suele confundirse y equipararse con otros conceptos como el de creencias religiosas o religiosidad, así por ejemplo, se hace notar en algunos de los estudios previamente comentados el empleó del término "fe religiosa" como equivalente al término "espiritualidad"(12,22,23).

Varios autores coinciden al definir la espiritualidad, como una búsqueda personal, que se caracteriza por un sentimiento de integración con la vida y el mundo, que se desarrolla para entender la existencia humana, su significado desde la individualidad, y la relación con lo sagrado o trascendente; que puede o no conducir al desarrollo de rituales religiosos y a la formación de una comunidad $^{(4,5,8,12)}$. Mientras que la religión, es un sistema organizado de creencias, prácticas, rituales, y símbolos que caracteriza a una comunidad ${ }^{(4,12,14)}$. Se considera que la religión está compuesta por tres dimensiones $^{(4)}$ : una cognitiva, con relación a las creencias religiosas; otra conductual que comprende comportamientos religiosos y rituales, institucionalizados y/o convencionales; y la afectiva, que corresponde a los vínculos entre el hombre y la trascendencia.

De la religión se desprende la religiosidad, la cual surge como una forma de expresión Ilevada a la conducta, la cuál parte del sistema de creencias y cultos organizados; conformada por comportamientos, ritos, normas y valores. Tiene por objetivo dirigir la vida de aquellas personas interesadas en vincularse con lo divino. La religiosidad da por lo tanto, un carácter directivo a la persona, ya que la dota de los conocimientos necesarios para acercarse y buscar aquello que considere divino, a través del adoctrinamiento y la congregación con otros ${ }^{(12,14)}$.
Como puede observarse, existen diferencias en los constructos de religión y espiritualidad; donde la religión es vista como sustantiva, estática, institucional y objetiva; y la espiritualidad es evaluada como funcional, personal, subjetiva, basada en la experiencia ${ }^{(4,8)}$. Sin embargo, en lo cotidiano constantemente ambos términos se utilizan como sinónimos. Es importante entonces, conocer cuál es el concepto de espiritualidad que tienen los profesionales de la salud(24).

Ante esto, se han realizado diversas investigaciones respecto al significado de la espiritualidad con profesionales de la salud; una de estas consistió en un estudio descriptivo, con el objetivo de conocer qué entienden los profesionales hospitalarios por espiritualidad y su abordaje, emplearon cuestionarios autoadministrados. Concluyeron que la espiritualidad no es una cuestión de interés para los profesionales; para la mayoría es una necesidad de esperanza, una búsqueda de sentido. Los participantes de la investigación descrita, consideraron que existe una formación insuficiente, a pesar de su necesidad en el abordaje con el paciente ${ }^{(22)}$.

Así mismo, se realizó un estudio observacional, descriptivo y transversal con una muestra de diferentes profesionales que laboraban en cuidados paliativos. Para la recolección de datos emplearon un cuestionario abierto. Concluyeron que los constructos espiritualidad, religión y búsqueda de sentido no quedan muy claros para los participantes del estudio. Propusieron como posible explicación, que los profesionales de cuidados paliativos confunden estos términos; y que existen, por lo tanto, diferencias conceptuales entre las necesidades espirituales y las religiosas, y que a su vez, estas diferencias pueden condicionar las propuestas de intervención ${ }^{(24)}$.

Por otra parte, fue realizado un estudio con el objetivo de comprender cómo los 
profesionales de la salud le dan significado a la religiosidad y a la fe de los pacientes en tratamiento de cáncer, y cómo los profesionales perciben ese fenómeno. Encontraron que la mayoría de los profesionales entrevistados declararon ser miembros de alguna religión, entre ellas espiritualistas, católicos, y budistas, por mencionar algunas. Encontraron además, que los profesionales presentaban creencias relacionadas con la protección divina y reconocieron a la religiosidad como sustento y fuente de confort para el paciente y sus familiares, $y$ que por tanto facilita el enfrentamiento de la situación de enfermedad ${ }^{(23)}$.

Finalmente, se realizó un estudio, para explorar como las enfermeras entendían la espiritualidad y la procuración del cuidado espiritual. Les interesaba conocer el significado del cuidado espiritual, y cómo atendían las necesidades espirituales de sus pacientes. Llevaron a cabo una serie de entrevistas semiestructuradas en las que encontraron que para la mayoría de las participantes, el cuidado espiritual dependía de la práctica religiosa del paciente, y que estas profesionales incorporaban prácticas o creencias religiosas a los cuidados de enfermería. Así mismo, algunas de las enfermeras creyeron que el cuidado espiritual consiste en la integración de prácticas religiosas al cuidado físico y psicológico ${ }^{(20)}$.

En síntesis, la espiritualidad, es una necesidad fundamental en el cuidado paliativo, sin embargo la literatura reporta que existen inconsistencias en cuanto a la definición de los términos "espiritualidad" y "religiosidad", lo que genera confusión en su abordaje, es decir, al no tener claro qué es la espiritualidad para el equipo multidisciplinario, no se puede proponer un plan de atención para el paciente que implique un cuidado espiritual. Para poder sensibilizar al personal sanitario sobre la importancia de la proporción y participación en el cuidado espiritual para el paciente, debemos conocer qué es lo que los profesionales de salud entienden por espiritualidad. Por lo tanto, esta investigación tiene por objetivo conocer el concepto de espiritualidad y religiosidad que tienen los profesionales de salud de una unidad de cuidados paliativos.

\section{MÉTODO}

Fue un estudio prospectivo, no experimental transversal, de tipo descriptivo; con un muestreo no probabilístico de sujetos voluntarios.

\section{Participantes}

Aquellos profesionales que aceptaron participar y firmaron el consentimiento informado, obteniendo la participación de 34 profesionales (personal adscrito y en formación) de las diferentes áreas que conforman la Unidad de Cuidados Paliativos del Instituto Nacional de Cancerología.

\section{Procedimiento}

Los datos se recabaron a través de la técnica de redes semánticas naturales ${ }^{(25,26)}$, con la cual se analizó el significado de los conceptos de espiritualidad y religiosidad. La técnica consistió en la ejecución de dos tareas:

1) Escribir 10 palabras que definan, con la mejor claridad y precisión, el concepto que se les presenta (pueden ser verbos, adverbios, sustantivos, adjetivos, etc. sin utilizar artículos y preposiciones).

2) La jerarquización de las palabras definidoras, asignando el número 10 a la palabra que mejor defina el concepto, el 9 a la que le sigue en importancia, así sucesivamente hasta asignar el número 1 a la de menor importancia. 
La aplicación se realizó dentro de la unidad de forma individual, proporcionándoles un cuadernillo, que constó de una ficha de identificación, instrucciones para su realización, y dos palabras estímulo (Espiritualidad y Religiosidad). Para aquellos participantes que no se encontraban en la unidad se les envió un consentimiento informado y un cuadernillo por correo electrónico.

\section{Análisis estadístico}

Con base en las frecuencias y la jerarquización de palabras asociadas a espiritualidad y religiosidad, se obtuvieron los siguientes valores:

El valor J, que es el resultado de la cantidad de palabras definidoras totales. El valor $M$, también Ilamado peso semántico, a partir de este valor se obtuvo el conjunto $S A M$, que corresponde al grupo de las 15 palabras definidoras con mayor $M$.

El valor FMG que es el indicador de la distancia semántica; que representa en forma de porcentaje a las palabras definidoras que se encuentran más cercanas al estímulo.

\section{RESULTADOS}

La muestra de 34 profesionales estuvo integrada por: 21 médicos, 3 enfermeras (o), 5 psicólogas, 2 nutriólogas, y 3 trabajadoras sociales. De los cuáles el 29\% eran hombres entre 23 y 45 años, con una media de edad de 26 años; y el 70\% fueron mujeres entre 22 y 56 años, con una media de edad de 33 años. El 62\% reportó ser de religión católica, 3\% de religión judía, y un 23\% reportó ninguna.

Dentro de los valores obtenidos, el valor $\mathrm{J}$, presenta diferencias en el total de palabras generadas para ambos conceptos; mostrando un puntaje mayor en las palabras generadas para el estímulo de religiosidad.
Valor J: Espiritualidad = 154; Religiosidad $=169$.

Así mismo, se encontró que los participantes definieron el estímulo de espiritualidad como: paz, fe, dios, creencia, alma, amor, tranquilidad, religión, armonía, esperanza, meditación, bondad, espíritu, trascendencia, creencias (ver la tabla 1).

En cuanto al estímulo de religiosidad, fue definida como: fe, dios, iglesia, esperanza, creencia, creencias, ritos, religión, amor, fanatismo, espiritualidad, compromiso, oración, reglas, sacerdote (ver la tabla 2).

Así también, se encontró que los porcentajes de las palabras definidoras que se encuentran más cercanas al estímulo de espiritualidad son: paz (100\%), fe $(88 \%)$, dios $(74 \%)$, creencia $(60 \%)$, alma (57\%); y para el estímulo religiosidad son: fe $(100 \%)$, dios $(80 \%)$, iglesia $(44 \%)$ ) ver figuras 1 y 2).

Respecto a las diferencias entre las disciplinas, se obtuvieron los valores del subgrupo de los médicos, ya que representan la mayor parte de la muestra. Obteniendo el valor J: espiritualidad $=113$; religiosidad $=121$; el cual reporta un puntaje mayor para el estímulo de religiosidad. De igual forma se encontraron similitudes en el conjunto SAM para ambas palabras estímulo. Se destacan, sin embargo, diferencias en algunas de las palabras asociadas a ambos estímulos, aunque con mayor distancia semántica del significado; que para el estímulo de espiritualidad son: ayudar e interiorización; y para el estímulo de religiosidad son: moral y rezar (ver tablas 3 y 4).

Finalmente se obtuvo el valor FMG; presentando las principales palabras definidoras, para espiritualidad: paz (100\%), fe $(84 \%)$, alma $(68 \%)$, dios $(66 \%)$, creencia $(56 \%)$; y para religiosidad: fe $(100 \%)$, dios (93\%), iglesia (59\%), religión (53\%) (ver las figuras 3 y 4 ). 
Tabla 1. Valores obtenidos de la muestra total para el estímulo Espiritualidad

\begin{tabular}{|c|c|c|}
\hline Valor $\mathbf{M}$ & Conjunto SAM & FMG \\
\hline 103 & Paz* $^{*}$ & $100 \%$ \\
\hline 91 & Fe & $88 \%$ \\
\hline 77 & Dios & $74 \%$ \\
\hline 63 & Creencia & $60 \%$ \\
\hline 60 & Alma* & $57 \%$ \\
\hline 48 & Amor & $46 \%$ \\
\hline 46 & Tranquilidad* & $44 \%$ \\
\hline 41 & Religión & $39 \%$ \\
\hline 38 & Armonía* & $36 \%$ \\
\hline 36 & Esperanza $^{*}$ & $34 \%$ \\
\hline 33 & Meditación* & $31 \%$ \\
\hline 28 & Bondad* $^{*}$ & $26 \%$ \\
\hline 27 & Espíritu* $^{*}$ & $25 \%$ \\
\hline 25 & Trascendencia* & $23 \%$ \\
\hline 25 & Creencias & $23 \%$ \\
\hline
\end{tabular}

*Diferencias en comparación con el estímulo de religiosidad.

Tabla 2. Valores obtenidos de la muestra total para el estímulo Religiosidad.

\begin{tabular}{|c|c|c|}
\hline Valor M & Conjunto SAM & FMG \\
\hline 122 & Fe & $100 \%$ \\
\hline 98 & Dios & $80 \%$ \\
\hline 54 & Iglesia* & $44 \%$ \\
\hline 44 & Esperanza & $36 \%$ \\
\hline 42 & Creencia & $34 \%$ \\
\hline 41 & Creencias & $33 \%$ \\
\hline 40 & Ritos* & $32 \%$ \\
\hline 37 & Religión & $30 \%$ \\
\hline 36 & Amor & $29 \%$ \\
\hline 29 & Fanatismo* & $23 \%$ \\
\hline 27 & Espiritualidad* $^{*}$ & $21 \%$ \\
\hline 27 & Compromiso* $^{*}$ & $21 \%$ \\
\hline 24 & Oración* & $19 \%$ \\
\hline 23 & Reglas* & $18 \%$ \\
\hline 23 & Sacerdote & $18 \%$ \\
\hline
\end{tabular}

*Diferencias en comparación con el estímulo de espiritualidad. 
Figura 1. Valores FMG obtenidos de la muestra total para el estímulo Espiritualidad.

\section{ESPIRITUALIDAD}

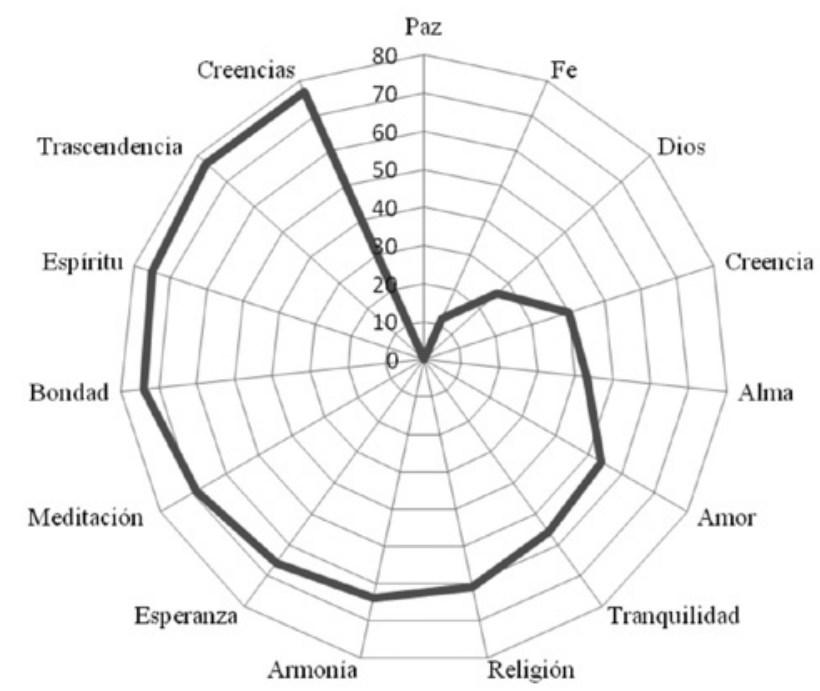

Figura 2. Valores obtenidos de la muestra total para el estímulo Religiosidad.

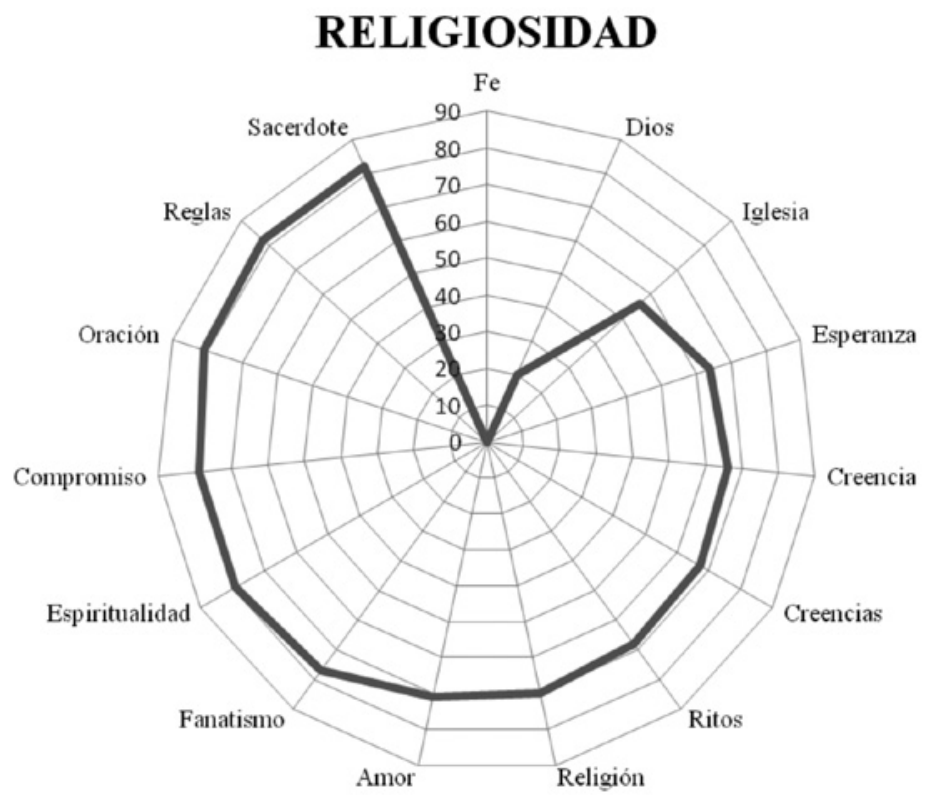


Tabla 3. Valores obtenidos del subgrupo de médicos para el estímulo Espiritualidad.

\begin{tabular}{|c|c|c|}
\hline Valor $\mathbf{M}$ & Conjunto SAM & FMG \\
\hline 70 & Paz & $100 \%$ \\
\hline 59 & Fe & $84 \%$ \\
\hline 48 & Alma & $68 \%$ \\
\hline 47 & Dios & $66 \%$ \\
\hline 40 & Creencia & $56 \%$ \\
\hline 33 & Tranquilidad & $46 \%$ \\
\hline 31 & Religión & $43 \%$ \\
\hline 27 & Amor & $37 \%$ \\
\hline 25 & Meditación & $34 \%$ \\
\hline 23 & Esperanza & $31 \%$ \\
\hline 23 & Trascendencia & $31 \%$ \\
\hline 21 & Bondad & $28 \%$ \\
\hline 20 & Ayudar* & $27 \%$ \\
\hline 19 & Armonía & $26 \%$ \\
\hline 16 & Interiorización* & $22 \%$ \\
\hline
\end{tabular}

*Diferencias en comparación con el estímulo de espiritualidad de la muestra total.

\section{Tabla 4. Valores obtenidos del subgrupo de médicos para el estímulo Religiosidad.}

\begin{tabular}{|c|c|c|}
\hline Valor $\mathbf{M}$ & Conjunto SAM & FMG \\
\hline 70 & Fe & $100 \%$ \\
\hline 65 & Dios & $93 \%$ \\
\hline 41 & Iglesia & $59 \%$ \\
\hline 37 & Religión & $53 \%$ \\
\hline 34 & Ritos & $49 \%$ \\
\hline 28 & Creencia & $40 \%$ \\
\hline 27 & Espiritualidad & $38 \%$ \\
\hline 24 & Esperanza & $34 \%$ \\
\hline 23 & Sacerdote & $32 \%$ \\
\hline 22 & Moral* & $31 \%$ \\
\hline 20 & Creencias & $28 \%$ \\
\hline 20 & Reglas & $28 \%$ \\
\hline 19 & Rezar* & $27 \%$ \\
\hline 18 & Amor & $25 \%$ \\
\hline 18 & Compromiso & $25 \%$ \\
\hline
\end{tabular}

*Diferencias en comparación con el estímulo de religiosidad de la muestra total. 
Figura 3. Valores FMG obtenidos del subgrupo de médicos para el estímulo Espiritualidad.

\section{ESPIRITUALIDAD}

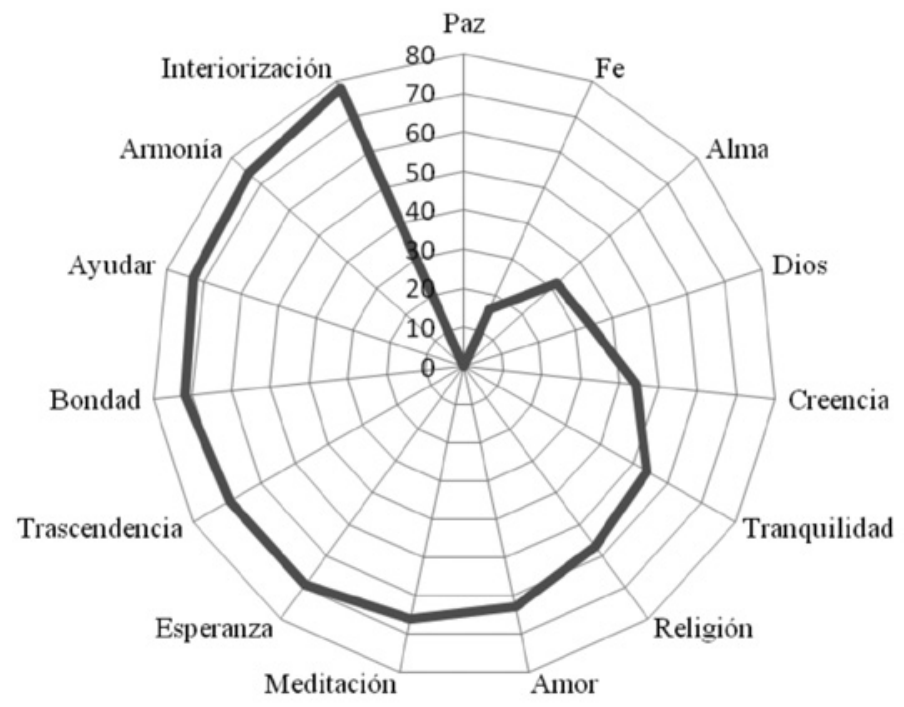

Figura 4. Valores FMG obtenidos del subgrupo de médicos para el estímulo Religiosidad.

\section{RELIGIOSIDAD}

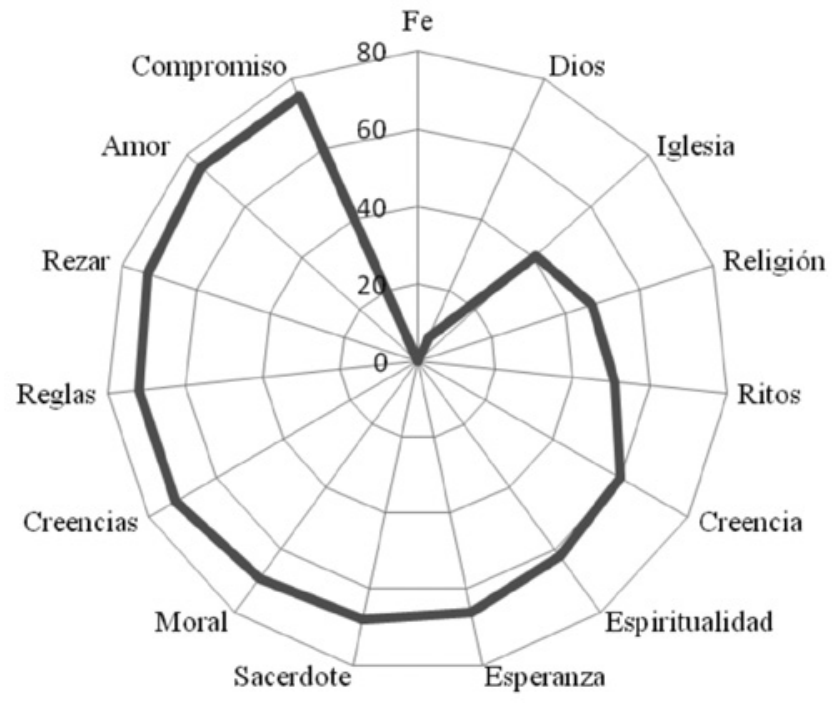




\section{DISCUSIÓN}

De acuerdo a los resultados reportados, se puede apreciar una mayor cantidad de palabras asociadas al estímulo religiosidad, lo cual puede estar relacionado con el porcentaje de profesionales participantes de religión católica, quienes generaron por tanto, palabras comunes en su conocimiento o práctica religiosa. Así mismo se pueden observar grandes semejanzas en las palabras asociadas a la definición de ambos estímulos, tales como: fe, dios, creencia, amor, religión, esperanza, creencias; lo cual coincide con lo referido en investigaciones anteriores con relación a la dificultad de los profesionales de cuidados paliativos para distinguir ambos conceptos, lo que lleva a confundir las necesidades espirituales de las religiosas al abordar al paciente ${ }^{(24)}$.

Sin embargo, se presentan, diferencias importantes en las definiciones de ambos estímulos; asignando al concepto de espiritualidad las palabras: Paz, alma, tranquilidad, armonía, meditación, bondad, espíritu, trascendencia; las cuales parecen estar relacionadas con una búsqueda personal; lo que coincide con lo propuesto por varios autores al definir la espiritualidad como, la experiencia de un sentimiento de integración con la vida y el mundo, con el objetivo de entender la existencia humana $^{(4,5,8,12),}$. En comparación con las palabras asignadas al concepto de religiosidad: Iglesia, ritos, fanatismo, espiritualidad, compromiso, oración, reglas, sacerdote; las cuáles muestran palabras relacionadas con formas de expresión, conductas, normas y valores presentes en personas con un interés aparente de vincularse con aquello que consideren divino, lo que pudo ser adquirido por una doctrina en particular o con el objetivo de convivir en comunidad ${ }^{(12,14)}$.

Con respecto a los resultados del subgrupo de los médicos, destacan diferencias en algunas de las palabras aso- ciadas a ambos estímulos, aunque estas presentan una mayor distancia semántica, manifiestan la noción de reconocimiento de atención de las necesidades espirituales, asociado a el proceso personal que implica para el profesional enfrentar dichas necesidades ${ }^{(4,5,8,12,13,19,20)}$. Así también se muestran palabras asociadas a conductas o a un conjunto de valores relacionados con una comunidad o religión en común; determinando el comportamiento de sus seguidores $^{(12,14)}$; lo cual puede estar influyendo en el abordaje de las necesidades espirituales por parte de los profesionales.

Se puede apreciar que los conceptos de espiritualidad y religiosidad resultan confusos para el equipo de cuidados paliativos; se identificó, sin embargo, que los profesionales distinguen ambos conceptos; lo que puede sugerir que los profesionales en cuidados paliativos, cuentan con recursos personales para abordar estas necesidades, pero a la vez carecen de información y entrenamiento para su atención, así como de propuestas de atención de las necesidades espirituales. Existen propuestas que sugieren que todos los miembros del equipo de cuidados paliativos deberían recibir entrenamiento en cuidados espirituales de acuerdo a su grado de intervención con los pacientes; así como tener los conocimientos sobre las opciones disponibles para abordar la espiritualidad de los pacientes, lo que incluye información y recursos espirituales ${ }^{(1)}$.

Se identificó, además, que la religiosidad, influye en la atención de las necesidades espirituales, a partir de los conocimientos, conductas y valores que poseen los profesionales respecto a la religión que profesan o comunidad a la que pertenecen. Así también, encontramos que la espiritualidad implica para esta población, paz, es decir, un proceso personal, de contacto y equilibrio con su entorno; de lo que se puede inferir que los profesionales de la unidad contemplan la posibilidad de 
experimentar esta definición en su práctica clínica diaria, o bien que cuentan con noción de la misma a nivel personal.

Resulta, entonces, de gran importancia analizar los recursos personales y de formación con los que cuentan los profesionales para identificar y atender las necesidades espirituales de los pacientes y sus familias en fases avanzadas de enfermedad, además de la importancia de contar con entrenamiento y conocimiento sobre los modelos de atención de las necesidades espirituales existentes, basados en un contexto socio-cultural, pertinente a nuestra población.

\section{REFERENCIAS BIBLIOGRÁFICAS}

1. Puchalski C, Ferrellb B, Viranib R, OtisGreenb S, Bairdb P, Bull J et al. La mejora de la calidad de los cuidados espirituales como una dimensión de los cuidados paliativos: el informe de la Conferencia de Consenso; Estados Unidos, California: Sociedad Española de Cuidados Paliativos, Elsevier España, S.L.; 2011; 18:55-78.

2. VilaltaVilalta A. Evaluación de las necesidades espirituales de pacientes diagnosticados de cáncer avanzado y terminal. [Tesis Doctoral] Catalá:Facultat de Medicina, Departament de Cirugía, Universidad de Lleida, 2010.

3. Bermejo JC. Hospitalidad. Atendiendo el sufrimiento desde la hospitalidad, presencia y compasión, de las tradiciones de sabiduría clínica. En: IX Jornada Nacional SECPAL: Espiritualidad en la Clínica; Palma de Mallorca, 2011; p. 23-39.

4. Quinceno MJ, Vinaccia S. La salud en el marco de la psicología de la religión y la espiritualidad. Divers Perspect Psicol 2009;5:321-36.

5. Núñez P, Enriquez D, Irrázaval Ma. E. La espiritualidad en el paciente oncológico: Una forma de nutrir la esperanza y fomentar un afrontamiento positivo a la enfermedad. Ajayu, 2011;10:84-100.
6. Payán CE, Vinaccia S, Quinceno MJ, Cognición hacia la enfermedad, bienestar espiritual y calidad de vida en pacientes con cáncer en estado terminal. Acta Colombiana de Psicología 2011;14:79-89.

7. Beca I, Juan P. Significado de la enfermedad y dignidad del enfermo [Internet]; 2007 [Acceso el 14 de febrero de 2013]. Disponible en: Medicina.udd.cl/centrobioetica/files/2010/10/significado_enfermedad.pdf

8. Navas C, Villegas H, Espiritualidad y Salud, Revista Ciencias de la Educación, 2006;1: 29-45

9. Peteet J. R., Spiritually integrated treatment of depression. A conceptual framework [Review]. Hindawi Publishing Corporation Depression Research and Treatment; 2012; 6. Doi: 10.1155/2012/124370

10. Sánchez Herrera B. Bienestar espiritual de enfermos terminales y de personas aparentemente sanas. Invest Educ Enferm 2009;27:86-95.

11. World Health Organization. WHO: Definition of palliative care, 2013.

12. Rivera-Ledesma, Montero-López Lena M., Ejercicio clínico y espiritualidad; An Psicol 2007;23:125-36.

13. Rodrigues Gomes A. M., La espiritualidad ante la proximidad de la muerte, Rev. Electrónica Trimestral de Enfermería, [Internet]. 2011 [Acceso el 12 de enero de 2013]; (22): 1-10. Disponible en: www. um.es/eglobal/

14. Secareccia D, Brown BJ. Impact of spirituality on palliative care physicians: personally and professionally. J Palliat Med 2009;12:805-9.Doi 10.1089/ jpm.2009.0038:

15. Balboni TA, Vanderwerker LC, Block S D, Paulk EM, Lathan CS, Peteet JR, et al. Religiousness and spiritual support among advanced cancer patients and associations with end-of-life treatment preferences and quality of life. J Clin Oncol 2007; 25:555-60. Doi: 10.1200/ JCO.2006.07.9046 
16. Stecca Battistella C. Sufrimiento existencial relacionado al bloqueo de las fuentes de sentido en la persona mayor paciente paliativa. Rev Dolor 2009;18:16-24.

17. Bertachini L, Pessini L, A importância da dimensão espiritual na práctica dos cuidados paliativos; Bioethikos, Centro Univsiário Sao Camilo 2010;4:315-23

18. RamióJofre A, Sant Joan De Deu E. Necesidades espirituales y atención profesional. En: IX Jornada Nacional SECPAL: espiritualidad en la clínica; Palma de Mallorca, 2011; 52-4.

19. Fernández N, Naudeillo M, Escola A, Dolorsquera Saiz N, CssMutuam Güell. Reflexión sobre creencias espirituales de los profesionales en una unidad de cuidados paliativos. En: IX Jornada Nacional SECPAL: espiritualidad en la clínica; Palma de Mallorca, 2011; 79.

20. Dhamani KA, Paul P, Olson JK. Tanzanian nurses understanding and practice of spiritual care. ISRN Nursing, 2011, 1-7. En línea. [Acceso 10 de abril de 2014] Disponible en: http://ecommons.aku.edu/ eastafrica_fhs_sonam/15

21. Barbero GJ, Prado RM, Rodríguez RR, Herrero BM, García LH, Rodríguez de la Rúa A. Aportaciones cualitativas a un grupo de encuestados sobre la dimensión espiritual.
En: IX Jornada Nacional SECPAL: espiritualidad en la clínica; Palma de Mallorca, $2011 ; 85$.

22. Gómez Cano S, Herrador Fuentes B, Fernández $R$, ¿Es necesario abordar la espiritualidad? ¿Qué opinan nuestros profesionales sanitarios? En: IX Jornada Nacional SECPAL: espiritualidad en la clínica; Palma de Mallorca, 2011; 89.

23. Espíndula JA, Martins Do Valle ER, Bello AA. Religión y espiritualidad: una perspectiva de profesionales de la salud; Latino-Am Enfermagem 2010;1229-36.

24. Soler Labajos E, Viel Sirito S, Soriano Pujol D, Arenas López U, Sanitari del Garraf C. ¿Qué piensan los profesionales de cсpp acerca de la atención espiritual y religiosa al final de la vida? En: IX Jornada Nacional SECPAL: espiritualidad en la clínica; Palma de Mallorca, 2011; 110.

25. Vera Noriega JA, Pimentel CE, Batista de Albuquerque FJ, Redes semánticas: aspectos teóricos, técnicos, metodológicos y analíticos; RaXimhai, Univ. Autónoma Indígena de México 2005;1:439-51.

26. Valdez Medina JL. Técnica de redes semánticas. En: las redes semánticas naturales, usos y aplicaciones en psicología social, Universidad Autónoma del Estado de México; 2002. p. 65-80. 